\title{
An ultra-long and quite thin coronal loop without significant expansion
}

\author{
Dong $\mathrm{Li}^{1,2}$, Ding Yuan ${ }^{3 \star}$, Marcel Goossens ${ }^{4}$, Tom Van Doorsselaere ${ }^{4}$, Wei $\mathrm{Su}^{5}$, Ya Wang ${ }^{1}$, Yang $\mathrm{Su}^{1,6}, \&$ \\ Zongjun Ning ${ }^{1,6}$ \\ 1 Key Laboratory of Dark Matter and Space Astronomy, Purple Mountain Observatory, CAS, Nanjing 210033, PR China e-mail: \\ lidong@pmo.ac.cn \\ 2 State Key Laboratory of Space Weather, Chinese Academy of Sciences, Beijing 100190, PR China \\ ${ }^{3}$ Institute of Space Science and Applied Technology, Harbin Institute of Technology, Shenzhen 518055, PR China e-mail: \\ yuanding@hit.edu.cn \\ ${ }^{4}$ Centre for mathematical Plasma Astrophysics, Department of Mathematics, KU Leuven, Celestijnenlaan 200B, 3001 Leuven, \\ Belgium \\ 5 MOE Key Laboratory of Fundamental Physical Quantities Measurements, School of Physics, Huazhong University of Science and \\ Technology, Wuhan 430074, PR China \\ ${ }^{6}$ School of Astronomy and Space Science, University of Science and Technology of China, Hefei 230026, PR China
}

Received; accepted

\section{ABSTRACT}

Context. Coronal loops are the basic building blocks of the solar corona, which are related to the mass supply and heating of solar plasmas in the corona. However, their fundamental magnetic structures are still not well understood. Most coronal loops do not expand significantly, whereas the diverging magnetic field would have an expansion factor of about 5-10 over one pressure scale height.

Aims. In this study, we investigate a unique coronal loop with a roughly constant cross section, it is ultra long and quite thin. A coronal loop model with magnetic helicity is presented to explain the small expansion of the loop width.

Methods. This coronal loop was predominantly detectable in the $171 \AA \AA$ channel of the Atmospheric Imaging Assembly (AIA). Then, the local magnetic field line was extrapolated by a Potential-Field-Source-Surface model. Finally, the differential emission measure analysis made from six AIA bandpasses was applied to obtain the thermal properties of this loop.

Results. This coronal loop has a projected length of roughly $130 \mathrm{Mm}$, a width of about $1.5 \pm 0.5 \mathrm{Mm}$ and a lifetime of around 90 minutes. It follows an open magnetic field line. The cross section expanded very little (i.e., 1.5-2.0) along the loop length during its whole lifetime. This loop has a nearly constant temperature at about $0.7 \pm 0.2 \mathrm{MK}$, whereas its density exhibits the typical structure of a stratified atmosphere.

Conclusions. We use a thin twisted flux tube theory to construct a model for this non-expanding loop, and find that indeed with sufficient twist a coronal loop can attain equilibrium. However, we can not rule out other possibilities such as footpoint heating by small-scale reconnection, elevated scale height by a steady flow along the loop etc.

Key words. Sun: corona - Sun: UV radiation — Sun: magnetic fields — Sun: activity

\section{Introduction}

Coronal loops are the basic structures in the solar corona. They can be detected everywhere on the Sun, such as the quiet region, the active region, or the solar limb, and their size scale could be ranging from sub-Megameter to hundreds of Megameters in the lower corona. These coronal loops often confine plasmas at the temperature of Mega-Kelvin, so they are prominently detectable in the extreme ultraviolet (EUV) and X-ray bandpasses (Bray et al. 1991; Reale 2014). Moreover, the plasmas contained in a coronal loop may be either isothermal (e.g., Del Zanna, \& Mason 2003; Tripathi et al. 2009; Gupta et al. 2019) or multithermal (e.g., Schmelz \& Martens 2006; Kucera et al. 2019) along the

\footnotetext{
$\star$ Corresponding author
}

line of sight. In the corona, full-ionized plasma is frozen-in the magnetic field line. So the plasma properties are normally uniform along the loops, strong inhomogeneity are usually detected across the loops. By comparisons of the coronal imaging observations in EUV or X-ray channels, together with the extrapolated field lines derived from the photospheric magnetogram, the coronal loop was found to generally follow the magnetic field line (Poletto et al. 1975; Feng et al. 2007). That is, a closed coronal loop is usually consist of a loop apex and two footpoints rooted in two opposite polarities (e.g., Watko, \& Klimchuk 2000; Peter \& Bingert 2012), while an open coronal loop connects to one apparent polarity at the solar surface and extends radially into the heliosphere magnetic field (e.g., Gupta et al. 2019). Previous studies also suggested that the temperature variation along a 
coronal loop is highly sensitive to the heating mechanism (Priest et al. 1998; Warren et al. 2008). Therefore, to study the coronal loop in the complex magnetic environment could help us to better understand the fundamental problem in solar physics, i.e., coronal heating (e.g., Klimchuk 2000; Peter \& Bingert 2012; Li et al. 2015; Goddard et al. 2017).

The coronal loop is expected to expand with height, since the coronal magnetic field is found to diverge strongly with the height from the solar surface into the corona (Lionello et al. 2013; Chen et al. 2014). The expansion of coronal loop could be discovered on the active region (e.g., Malanushenko \& Schrijver 2013) or solar limb (e.g., Gupta et al. 2019). However, most of coronal loops observed in X-ray and EUV images are found to have roughly uniform widths in the plane of the sky, without significant expansions along their loop lengths, or only exhibit a small expansion from footpoints to the loop apex (e.g., Golub et al. 1990; Klimchuk et al. 1992; Klimchuk 2000; Watko, \& Klimchuk 2000; López Fuentes et al. 2006; Brooks et al. 2007; Kucera et al. 2019). The formation and appearance of these loops in the complex magnetic environment of the corona provides a pivotal test for a model of the coronal heating process (Klimchuk 2000; Petrie 2006; Peter \& Bingert 2012). On the other hand, the loop cross section carries the information of magnetic fields and the spatial distribution of corona heating, and the lower limit of the loop width is of fundamental importance to modern instrumentation, because it defines the spatial resolution of a spaceborne or ground-based telescope (Peter et al. 2013; Aschwanden $\&$ Peter 2017). Moreover, the loop width variation is a proxy of the inter-coupling of plasma dynamics and magnetic fields, so it is believed to play a key role in coronal heating (e.g., Vesecky et al. 1979; McTiernan \& Petrosian 1990; Mikić et al. 2013; Chastain \& Schmelz 2017). Aschwanden \& Peter (2017) find that the loop widths are marginally resolved in AIA images but are fully resolved in $\mathrm{Hi}-\mathrm{C}$ images, their model predicts a most frequent value at about $0.55 \mathrm{Mm}$.

The contradiction between the observed coronal loop with a roughly constant cross section and the extrapolated magnetic field with a strong expansion is still an open issue. In this paper, we investigated an ultra-long but quite thin coronal loop, which could be explained by a thin twisted flux tube model. The paper is organized as following: Section 2 introduces the data reduction and methods; and Section 3 describes properties of the coronal loop of interest; the conclusion and discussion are presented in Section 4.

\section{Data reduction and methods}

\subsection{Data reduction}

We combined the Atmospheric Imaging Assembly (AIA; Lemen et al. 2012) and the Helioseismic Magnetic Imager (HMI; Schou et al. 2012) on onboard the Solar Dynamic Observatory (SDO; Pesnell et al. 2012) to observe the active region NOAA 12524 near solar disk center (N20W04) on 2016 March 23. A unique coronal loop was predominately observed in the AIA $171 \AA$ channel, it is also vaguely simultaneously detectable in the AIA $193 \AA$ and $211 \AA$ channels, as shown in Figure 1 (a)-(c). This loop had an ultra long length and a very narrow width, which did not expand much radially along its length. Moreover, this coronal loop retained this form for about 90 minutes, as can be seen in the movie of anim.mp4. We then used the Potential-FieldSource-Surface (PFSS) model (Schrijver \& De Rosa 2003) to extrapolate the local magnetic field line. A cyan curve in Figure 1 (d) represents an open magnetic field line derived in the
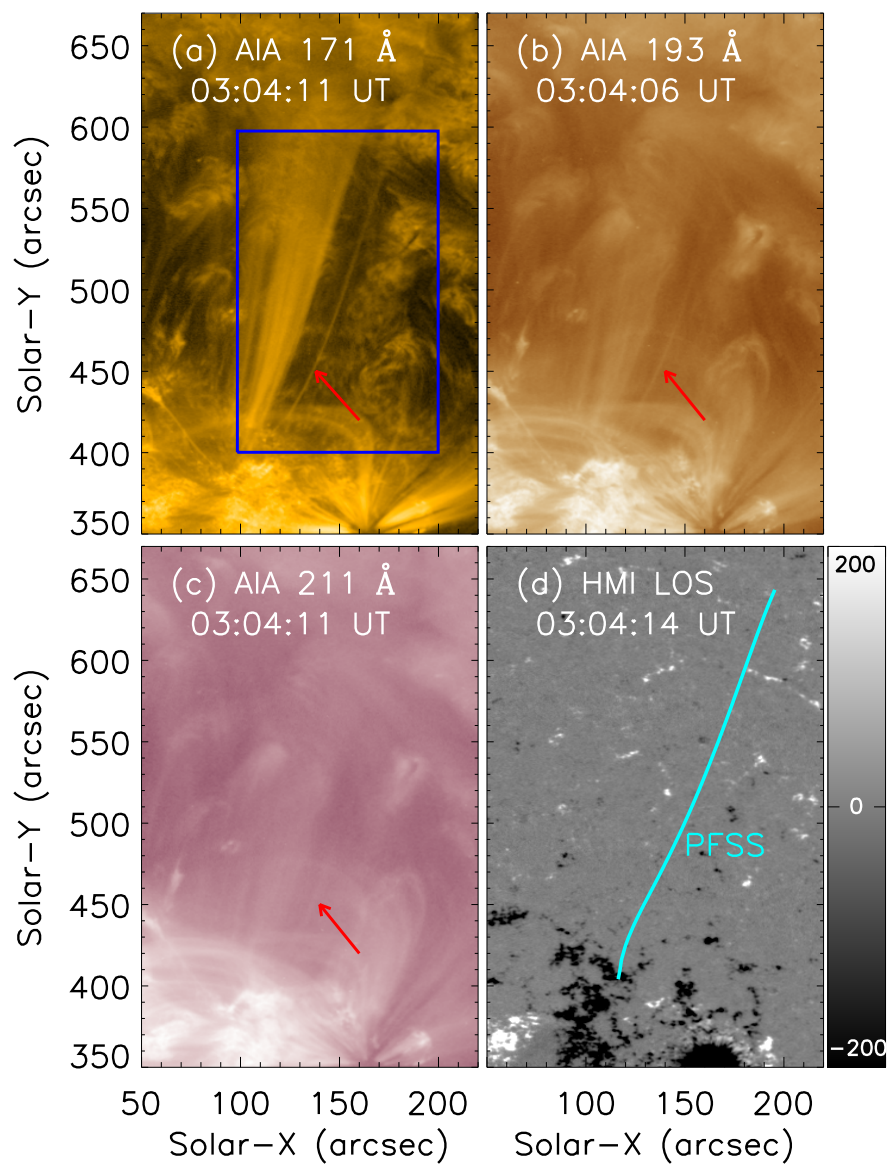

Fig. 1. Overview of the coronal loop on 2016 March 23. Field-of-view observed at about 03:04 UT in AIA $171 \AA$ (a), $193 \AA$ (b), $211 \AA$ (c) and HMI LOS magnetic magnetogram (d). The coronal loop of interest was indicated by an arrow in each panel. Within the PFSS magnetic field extrapolation, the magnetic field line closely aligned with this loop was overlaid (cyan curve) with the HMI LOS magnetogram. The region of interest used in the DEM analysis was enclosed in a blue rectangle. The evolution of this loop is shown in a movie of anim.mp4

PFSS model, which is closely aligned with the coronal loop of interest.

The SDO/AIA images used in this observation has a cadence of about 12 seconds, each pixel corresponds to about $0.6^{\prime \prime}$. The $\mathrm{SDO} / \mathrm{HMI}$ observes the full-disk photospheric magnetic fields. Both the AIA images and HMI magnetograms were calibrated with the standard routines in the Solar SoftWare package (Lemen et al. 2012; Schou et al. 2012).

\subsection{Loop Geometry}

This coronal loop was predominantly detectable in the AIA $171 \AA$ channel, therefore we used the AIA $171 \AA$ images to obtain its geometry. We created a two-dimension curvilinear coordinate, one curve coordinate is chosen to be aligned with the spine of the coronal loop, the second coordinate is set to be normal to the coronal loop. We made a bilinear interpolation of the emission intensity in the AIA $171 \AA$ channel into the curvilinear coordinate. Then each intensity profile across the loop was fitted with a Gaussian function plus a linear background. In order to improve the signal-to-noise ratio, we averaged three neigh- 


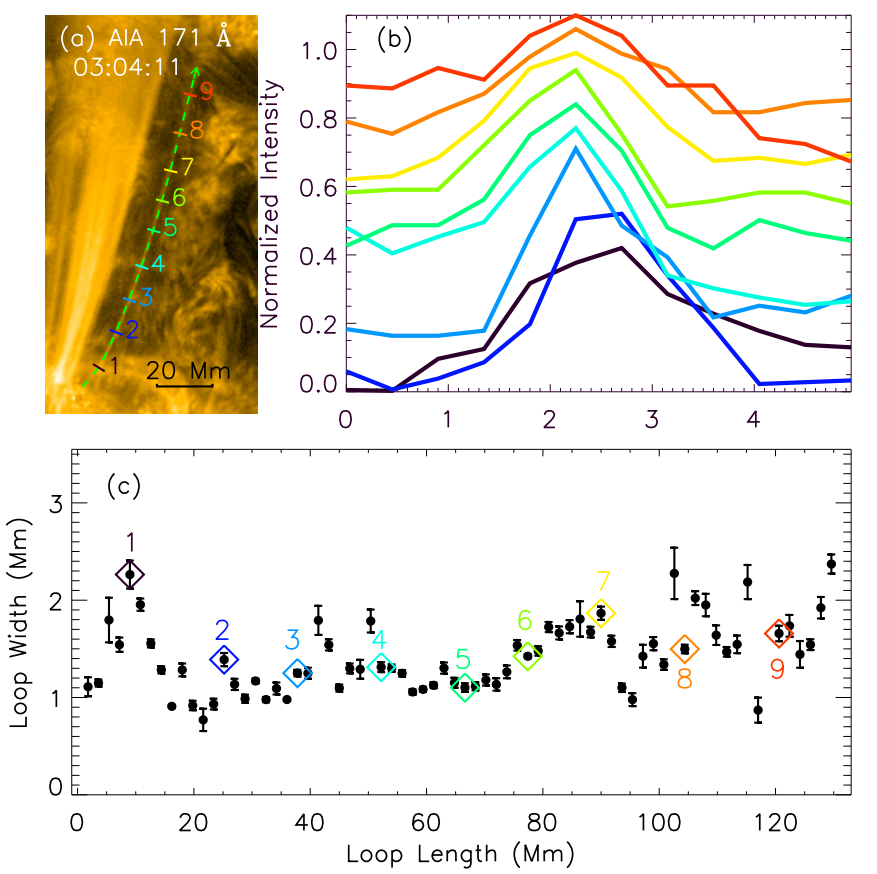

Fig. 2. Estimation of the loop width. (a) Smaller FOV $(\sim 76 \mathrm{Mm} \times 149 \mathrm{Mm})$ of AIA $171 \AA$ image. The loop is highlighted by a green arrow. Nine sample cuts was marked by short lines and numbered from 1 to 9 . (b) Intensity profiles along the cuts indicated in (a), which are normalized by their maximum intensity, respectively. The color used in each curve is the same as used in the numbered short lines in (a). Each profile is elevated progressively for visualization purpose. (c) Loop width variation along the loop length, the numbers mark the nine loop segments in panel (a).

boring profiles before fitting. The full width at half maximum (FWHM) of that Gaussian function was considered as the loop width $(w)$. The fitting error was used as uncertainties of the loop width (e.g., Aschwanden \& Boerner 2011; Gupta et al. 2019). The error for the AIA $171 \AA$ intensity was estimated according to the method described by Yuan, \& Nakariakov (2012).

In Figure 2 (a), nine cross cuts were made along the coronal loop and were plotted with the short color lines. Each intensity profile was scaled to a proper range and stacked in Figure 2 (b). The loop width was measured at locations perpendicular to the loop axis from the footpoint to the apparent top at a distance of about $130 \mathrm{Mm}$, as indicated in Figure 2 (c). We note that, at some positions (such as the positions close to ' 8 ' and ' 9 '), the obtained loop widths deviated significantly from those of their neighbors. The reason is that some random bright patchy background contaminated those emission intensity profiles. On the other hand, at some positions of (i.e., ' 1 ') the footpoint, the loop of interest had overlap with some bright closed loops, which resulted into the broad loop widths.

\subsection{DEM analysis}

In order to obtain the thermal properties of this loop, we focused on a smaller FOV as marked in Figure 1 (blue rectangle) and performed Differential emission measure (DEM) analysis. Observations taken from six EUV channels of SDO/AIA (94 $\AA$, $131 \AA, 171 \AA, 193 \AA, 211 \AA$, and $335 \AA$ ) were used to calculate the $\operatorname{DEM}(\mathrm{T})$ distribution for each pixel. We used an improved version (Su et al. 2018) of the sparse inversion code (Cheung et al. 2015). The derived solutions could provide valuable infor- mation by mapping the thermal plasma from 0.3 to $30 \mathrm{MK}$. The DEM uncertainties were estimated from Monte Carlo (MC) simulation ( $\mathrm{Su}$ et al. 2018). Random noise of the observed emission intensity was added to the MC simulation and the inversion was repeated for 100 times, then the standard deviations of the 100 MC simulations were used as the uncertainties of DEM solutions.

Figure 3 (a)-(d) draws the EM (Cheung et al. 2015; Su et al. 2018) maps from 0.32 MK to $3.98 \mathrm{MK}$, within which coronal loops are normally detected. These EM maps are calculated from a set of six re-binned AIA narrow-band maps with a pixel size of $1.2^{\prime \prime}$, in order to get clear view of the structures in different temperature ranges, whose emissions are accumulated along the line of sight (LOS) into the observed intensity. The coronal loop was clearly seen in the temperature range of $0.63 \mathrm{MK}$ to $1.12 \mathrm{MK}$ (b) and to a weaker extent in the $1.26 \mathrm{MK}-2.24 \mathrm{MK}$ range (c). In order to measure the temperature of the coronal loop of interest, we then plotted the DEM profiles (e) of seven positions (' $X$ ' in panel $b$ ) in the coronal loop. It can be seen that the obtained DEM profiles exhibit two peaks at about $0.8 \mathrm{MK}$ and $1.8 \mathrm{MK}$, respectively. However, the coronal loop of interest was most clearly seen in the DEM ranging from $0.63 \mathrm{MK}$ to 1.12 MK. So we suspect that the high-temperature peak at about 1.8 MK could originate from the emission of the diffuse background in the AIA $211 \AA$ channel. For a comparison, we then took the DEM profile of a reference point (' 0 ') at the background for cross-validation, as marked by the magenta ' + ' (Figure 3b). We noticed that the DEM profile (magenta) at the background indeed only had a prominent peak at about $1.8 \mathrm{MK}$.

The EM was calculated by integrating the DEM over temperatures, $\mathrm{EM}=\int \mathrm{DEM} \mathrm{d} T$. We only use the temperature ranges between $0.32-1.12 \mathrm{MK}$, this range is the effective temperature of the coronal loop of interest, as indicated by the grey region in Figure 3 (e). The EM could be considered to the product of the square of the number density of electrons $\left(n_{\mathrm{e}}\right)$ and LOS depth, which could be approximated with the loop width $(w)$. In this way, the electron number density could be calculated with $n_{\mathrm{e}}=\sqrt{\mathrm{EM} / w}$. Finally, a DEM-weighted mean temperature (such as $T=\int \mathrm{DEM} T \mathrm{~d} T / \int \mathrm{DEM} \mathrm{d} T$ ) is used to estimate the temperature of this coronal loop. The errors for the density and temperature were also calculated from the $100 \mathrm{MC}$ simulations. These steps were done for every pixel along the loop length. Then, using the obtained number density, plasma temperature and magnetic field, we calculated the plasma beta $(\beta)$ along the coronal loop.

\section{Properties of the coronal loop}

\subsection{Geometry of the loop}

The coronal loop under study was very thin and ultra long. It was detectable for a projected length of about $130 \mathrm{Mm}$. We note this as the lower limit, since it become diffuse and invisible in the background. The loop width was about $1 \mathrm{Mm}$ at the footpoint and expands to about $1.5 \mathrm{Mm}$ to $2.0 \mathrm{Mm}$ at the visible end. The loop expansion ratio was about 1.5 to 2.0. In this dataset of about 2 hours, we observed the distinctive coronal loop to fade out eventually, however, it had almost the constant width during its lifetime (see the movie of anim.mp4).

In the PFSS extrapolation model, we traced a magnetic field line that was closely aligned with the coronal loop of interest, as indicated by a cyan curve in Figure 1 (d). This magnetic field line was connected to a patch of negative polarity and extends to the outer space, therefore, this coronal loop could be regarded as 

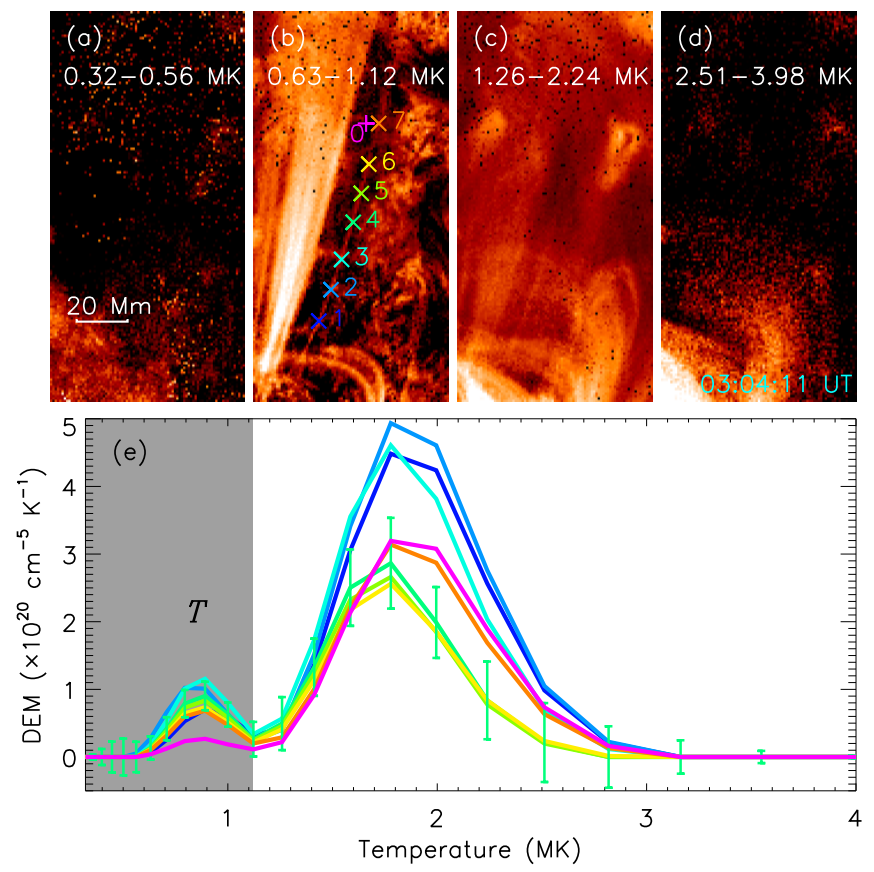

Fig. 3. DEM results of the target coronal loop. (a)-(d) Narrow band EM maps integrated in the temperature ranges of $0.32 \mathrm{MK}-0.56 \mathrm{MK}$, $0.63 \mathrm{MK}-1.12 \mathrm{MK}, 1.26 \mathrm{MK}-2.24 \mathrm{MK}$, and $2.51 \mathrm{MK}-3.98 \mathrm{MK}$, respectively. (e) DEM profiles at seven selected positions ('1'- '7') along the loop and one location (' 0 ') away from the loop, the color corresponds to the positions labeled in (b). For clarity, panel (e) only draws the error bars at the loop position ' 4 '. The grey region indicates the EM integrated range.

an open structure. It has an inclination in the range of $40^{\circ}-80^{\circ}$ based on the estimation in the PFSS model. The polarity at the loop footpoint has an average line-of-sight (LOS) magnetic field component of about 100 Gauss. Along the field line, the strength of the magnetic field is about 10 Gauss on average, whereas the maximum field strength could reach 60 Gauss. So we used 10 Gauss as the coronal loop's field strength.

\subsection{Thermal property of the loop}

Figure 3 presents the DEM results to the coronal loop of interest. It is apparent that this loop is most clearly identifiable in the EM map at $0.63 \mathrm{MK}-1.12 \mathrm{MK}$ (b). However, each DEM profile of the coronal loop normally has two peaks at around $0.8 \mathrm{MK}$ and $1.8 \mathrm{MK}(\mathrm{e})$, respectively. After a comparison with the background DEM profile (magenta), we concluded that the DEM peak at $1.8 \mathrm{MK}$ corresponds to a strong component from the emission of the diffuse background in the $211 \AA$ channel (Figure 1c). Therefore, we estimated that the coronal loop considered here had a temperature of about $0.8 \mathrm{MK}$.

In Figure 4, we present the quantitative estimation of the physical parameters of the coronal loop. Figure 4 (a) draws the AIA $171 \AA$ intensity (Yuan, \& Nakariakov 2012) and EM variations at the selected positions along the loop length. They first decreased quickly with the loop length and then became roughly stable. We notice that the AIA $171 \AA$ intensities are much stronger at the beginning, which could be due to the fact that some random bright patchy or closed loops contaminated at the base of the coronal loop (see also Figures 1 and 2).

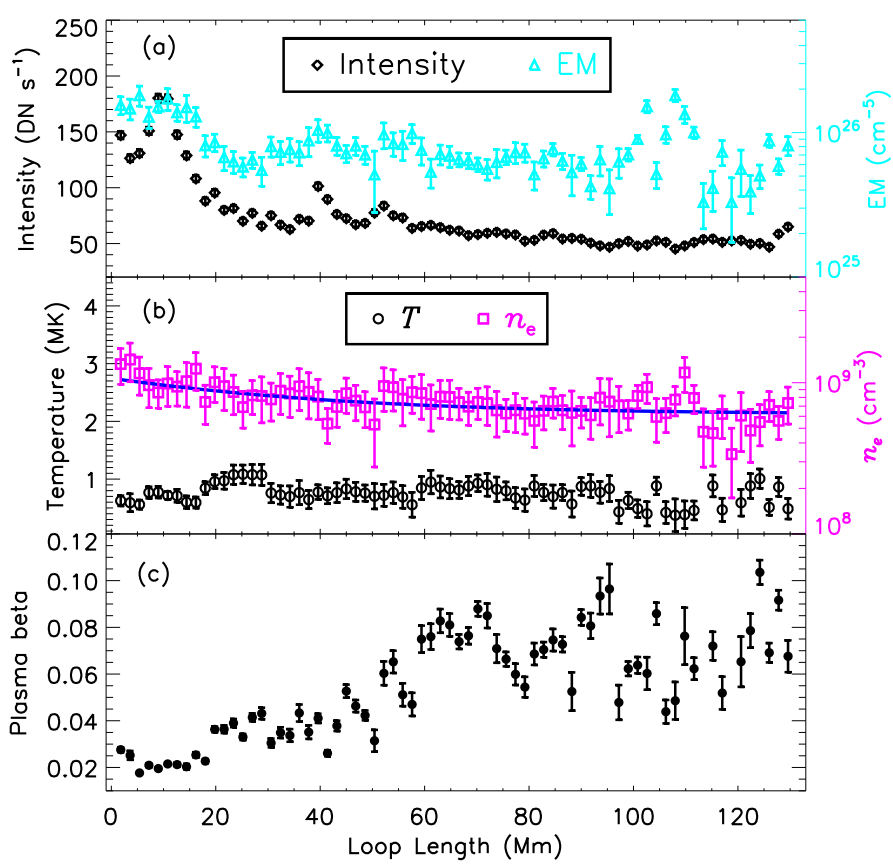

Fig. 4. Quantitative estimation of the coronal loop parameters. The EM (a), the emission intensity in the AIA $171 \AA$ bandpass (a), the plasma temperature (b), and the number density of electrons (b), as well as the plasma beta $(\beta)$ as a function of projected length along the loop. The blue line represents the best fitted result for the number density.

Figure 4 (b) plots the plasma temperature variation along the loop length. It shows that this coronal loop had a very uniform temperature at $(0.7 \pm 0.2) \mathrm{MK}$, this is consistent with our previous estimations. Since this loop was very thin, we cannot obtain the temperature distribution across the loop. Figure 4 (b) also shows the estimated number density of electrons along the loop length, with a mean number density of $(8 \pm 2) \times 10^{8} \mathrm{~cm}^{-3}$. The electron number density was about $1.0 \times 10^{9} \mathrm{~cm}^{-3}$ at the footpoint, and dropped off exponentially to around $5 \times 10^{8} \mathrm{~cm}^{-3}$ at the end of the loop. This was a pattern of stratification. Therefore, we fitted an exponential function to the density profile and obtained a density scale height of about $(38 \pm 13) \mathrm{Mm}$. This scale height only incorporate a reduced gravity, because this corona loop is inclined with respect to the solar radial. The theoretical density scale height is $(22.8 \pm 6.6) \mathrm{Mm}$ for a plasma with a temperature of $(0.7 \pm 0.2) \mathrm{MK}$. With the ratio of theoretical and fitted scale heights, we estimated that the loop deviated from the gravity vector on average by an angle of about $54^{\circ} \pm 30^{\circ}$. This value is consistent with the estimation of the extrapolated magnetic field, i.e., $40^{\circ}-80^{\circ}$.

Figure 4 (c) draws the plasma beta parameter $(\beta)$ as a function of the loop length. It can be seen that the plasma beta increased from about 0.02 at the footpoint to roughly 0.1 at its visible end. The average plasma beta of this loop is estimated to be $\sim 0.056 \pm 0.037$.

\section{Conclusion and Discussion}

In this study, we used the SDO/AIA data to observe an open coronal loop associated with AR 12524. This coronal loops was clearly detected in AIA $171 \AA$ and to an weaked extent in the AIA $193 \AA$ and $211 \AA$ channels. This loop was ultra-long and had a small width, its lifetime was about 90 minutes. The loop 
width was about $1.5 \mathrm{Mm}$, and a projected length about 130 $\mathrm{Mm}$. The coronal loop investigated in this study is thinner and longer than those reported earlier. For instance, Aschwanden \& Boerner (2011) reported coronal loops of about 2-4 Mm wide and 10-40 Mm long. Moreover, the most loops has a lifetime of about 20-30 minutes (e.g., Peter \& Bingert 2012), whereas in our case, the loop survived for over one hour. The coronal loop had a plasma temperature of $(0.7 \pm 0.2) \mathrm{MK}$, no signification variation of temperature was detected along the loop. This loop is relatively cold (e.g., <1 MK) and is approximately uni-thermal, this is consistent with the spectroscopic and imaging observations (e.g., Del Zanna, \& Mason 2003; Warren et al. 2008; Tripathi et al. 2009). The electron number density was measured at about $1.0 \times 10^{9} \mathrm{~cm}^{-3}$, and it dropped off exponentially to about $0.5 \times 10^{9} \mathrm{~cm}^{-3}$ at the visible end of the loop. This was a pattern of stratification, and then the density scale height was measured at roughly $(38 \pm 13) \mathrm{Mm}$. The plasma beta increases from about 0.02 to roughly 0.1 , it means that the gas pressure decreases with height by a smaller amount than the magnetic pressure. More observational, geometrical and physical parameters of this loop are listed in table 1.

It should be mentioned that the little expansion was found in many coronal loops at SXR/EUV wavelengths since the eras of TRACE and earlier (e.g., Klimchuk et al. 1992; Klimchuk 2000; Watko, \& Klimchuk 2000; Peter \& Bingert 2012; Kucera et al. 2019). These coronal loops were closed structures and often exhibited weak expansion from double footpoints to the loop-apex (López Fuentes et al. 2006; Brooks et al. 2007). Although an open coronal loop of about $280 \mathrm{Mm}$ long was reported by Gupta et al. (2019), but its loop width expanded from $20 \mathrm{Mm}$ at the footpoint to $80 \mathrm{Mm}$ at the loop top. Here, we investigate an ultralong $(\sim 130 \mathrm{Mm})$ and very thin $(\sim 1.5 \pm 0.5 \mathrm{Mm})$ coronal loop, which does not appear to undergo significant expansion as it rises above the photosphere.

A very interesting point is that the loop width expanded only by a factor of about 1.5 to 2.0, whereas this loop had a projected length of about three pressure scale heights. The pressure has decreased by $95 \%$ (i.e, $1-e^{-3}$ ), the cross-section of this loop should expand by a factor of about 19 . However, this is not supported by the observation, therefore, to explain the nearly constant cross-section of this coronal loop, there must be unresolved features (e.g., Klimchuk et al. 2000; Petrie 2008). This steady coronal loop has a lifetime much longer than the timescales of the radiative cooling and thermal conduction, therefore, we used a magnetostatic model (appendix A) to explain the small radial expansion of this loop. We used a thin flux tube approximation and assumed the magnetic field has a twist term (e.g., Ferriz-Mas \& Schüssler 1989). Only when the twist term was large enough, then the external pressure became negative. Thus, we could find an upper limit for the twist, the detailed derivation process was seen in the appendix B. We then constrained the expansion factor to be 1.5 and obtained the solution for the external pressure. We could see that to constrain the thin magnetic flux tube, the twist has to be smaller than about 0.65 (see, Figure B.1). Therefore, with a sufficiently twisted magnetic field, this coronal loop could be constrained at magnetic equilibrium state.

If we go beyond a magnetostatic equilibrium, this loop has to be supplied with steady flows of mass and energy, as we did not observe any features propagating emission intensity or brightening. This flow must have very long lifetime or should occur intermittently with short time scales. Evidence has collected that the footpoint of a coronal loop could have an unresolved minority-polarity, an miniature bipolar field emerging into a constant background field. This configuration favors small scale magnetic reconnection (Wang et al. 2019). Repetitive reconnections provide continuous mass and energy to the coronal loops (Chitta et al. 2018). Unfortunately we did not observe features to support this scenario, however we shall note that it could be that the AIA instrument is not sensitive enough to capture these features.

At last, we stress that the loop widths are measured as the FWHM of the cross-sectional profiles of the coronal loop detected in the SDO/AIA $171 \AA$ channel. One AIA pixel corresponds to $0.44 \mathrm{Mm}$ (Lemen et al. 2012). A loop width of about 1.5 to $2.0 \mathrm{Mm}$ spans about 4-5 pixels. This measurement is obtained by fitting a intensity profile with about 12 pixels, this practice could reach a higher accuracy than the pixel scale, and has been used by many other researchers (e.g., Aschwanden \& Boerner 2011; Anfinogentov et al. 2013; Anfinogentov \& Nakariakov 2019; Reale 2014).

Acknowledgements. We acknowledge the anonymous referee for his/her valuable comments. This study is supported by NSFC under grant 11973092, 11803008, 11873095, 11790300, 11790302, 11729301, 11773079, the Youth Fund of Jiangsu No. BK20171108 and BK20191108. The Laboratory No. 2010DP173032. D.L. is also supported by the Specialized Research Fund for State Key Laboratories. D.Y. is supported by the NSFC grant 11803005, 11911530690, Shenzhen Technology Project (JCYJ20180306172239618), and Shenzhen Science and Technology program (group No. KQTD20180410161218820). T.V.D. is supported by the European Research Council (ERC) under the European Union Horizon 2020 research and innovation programme (grant agreement No 724326) and the C1 grant TRACEspace of Internal Funds KU Leuven (number C14/19/089). Y.S. is supported by the NSFC grant U1631242, 11820101002, and the Jiangsu Innovative and Entrepreneurial Talents Program.

\section{References}

Anfinogentov, S., Nisticò, G., \& Nakariakov, V. M. 2013, A\&A, 560, A107 Anfinogentov, S. A., \& Nakariakov, V. M. 2019, ApJ, 884, L40 Aschwanden, M. J., \& Boerner, P. 2011, ApJ, 732, 81

Aschwanden, M. J., \& Peter, H. 2017, ApJ, 840, 4

Bray, R. J., Cram, L. E., Durrant, C., et al. 1991, Plasma Loops in the Solar Corona

Brooks, D. H., Warren, H. P., Ugarte-Urra, I., et al. 2007, PASJ, 59, S691 Chastain, S. I., \& Schmelz, J. T. 2017, arXiv e-prints, arXiv:1705.06776 Chen, F., Peter, H., Bingert, S., et al. 2014, A\&A, 564, A12

Cheung, M. C. M., Boerner, P., Schrijver, C. J., et al. 2015, ApJ, 807, 143 Chitta, L. P., Peter, H., \& Solanki, S. K. 2018, A\&A, 615, L9 Del Zanna, G., \& Mason, H. E. 2003, A\&A, 406, 1089

Dudík, J., Dzif̌ćáková, E., \& Cirtain, J. W. 2014, ApJ, 796, 20

Feng, L., Inhester, B., Solanki, S. K., et al. 2007, ApJ, 671, L205

Ferriz-Mas, A., \& Schüssler, M. 1989, Geophysical and Astrophysical Fluid Dynamics, 48, 217

Golub, L., Herant, M., Kalata, K., et al. 1990, Nature, 344, 842

Goddard, C. R., Pascoe, D. J., Anfinogentov, S., et al. 2017, A\&A, 605, A65

Gupta, G. R., Del Zanna, G., \& Mason, H. E. 2019, A\&A, 627, A62

Klimchuk, J. A., Lemen, J. R., Feldman, U., et al. 1992, PASJ, 44, L181

Klimchuk, J. A. 2000, Sol. Phys., 193, 53

Klimchuk, J. A., Antiochos, S. K., \& Norton, D. 2000, ApJ, 542, 504

Kucera, T. A., Young, P. R., Klimchuk, J. A., et al. 2019, ApJ, 885, 7

Lemen, J. R., Title, A. M., Akin, D. J., et al. 2012, Sol. Phys., 275, 17

Li, L. P., Peter, H., Chen, F., et al. 2015, A\&A, 583, A109

Lionello, R., Winebarger, A. R., Mok, Y., et al. 2013, ApJ, 773, 134

López Fuentes, M. C., Klimchuk, J. A., \& Démoulin, P. 2006, ApJ, 639, 459

Malanushenko, A., \& Schrijver, C. J. 2013, ApJ, 775, 120

McTiernan, J. M., \& Petrosian, V. 1990, ApJ, 359, 524

Mikić, Z., Lionello, R., Mok, Y., et al. 2013, ApJ, 773, 94

Peter, H., \& Bingert, S. 2012, A\&A, 548, A1

Peter, H., Bingert, S., Klimchuk, J. A., et al. 2013, A\&A, 556, A104

Petrie, G. J. D. 2006, ApJ, 649, 1078

Petrie, G. J. D. 2008, ApJ, 681, 1660

Pesnell, W. D., Thompson, B. J., \& Chamberlin, P. C. 2012, Sol. Phys., 275, 3

Poletto, G., Vaiana, G. S., Zombeck, M. V., et al. 1975, Sol. Phys., 44, 83

Priest, E. R., Foley, C. R., Heyvaerts, J., et al. 1998, Nature, 393, 545

Reale, F. 2014, Living Reviews in Solar Physics, 11, 4

Schmelz, J. T., \& Martens, P. C. H. 2006, ApJ, 636, L49

Schou, J., Scherrer, P. H., Bush, R. I., et al. 2012, Sol. Phys., 275, 229 
Table 1. Observational, geometrical and physical parameters of the analyzed coronal loop.

\begin{tabular}{ll}
\hline \hline Parameter & Value \\
\hline Date of observation & 2016 March 23 \\
Active region & NOAA 12524 \\
Projected length & $\sim 130 \mathrm{Mm}$ \\
Loop width & $1.5 \pm 0.5 \mathrm{Mm}$ \\
Expanded factor of loop width & $1.5-2.0$ \\
Plasma temperature & $0.7 \pm 0.2 \mathrm{MK}$ \\
Number density of electrons & $(8 \pm 2) \times 10^{8} \mathrm{~cm}^{-3}$ \\
Plasma beta & $0.056 \pm 0.037$ \\
Inclination angle of the extrapolated magnetic field & $40^{\circ}-80^{\circ}$ \\
Inclination angle inferred from stratified plasma & $54^{\circ} \pm 30^{\circ}$ \\
Fitted density scale height & $38 \pm 13 \mathrm{Mm}$ \\
Theoretical density scale height & $22.8 \pm 6.6 \mathrm{Mm}$ \\
\hline \hline
\end{tabular}

Schrijver, C. J., \& De Rosa, M. L. 2003, Sol. Phys., 212, 165

Su, Y., Veronig, A. M., Hannah, I. G., et al. 2018, ApJ, 856, L17

Tripathi, D., Mason, H. E., Dwivedi, B. N., et al. 2009, ApJ, 694, 1256

Vasheghani Farahani, S., Nakariakov, V. M., \& van Doorsselaere, T. 2010, A\&A,

517, A29

Vesecky, J. F., Antiochos, S. K., \& Underwood, J. H. 1979, ApJ, 233, 987

Watko, J. A., \& Klimchuk, J. A. 2000, Sol. Phys., 193, 77

Warren, H. P., Ugarte-Urra, I., Doschek, G. A., et al. 2008, ApJ, 686, L131

Wang, Y.-M., Ugarte-Urra, I., \& Reep, J. W. 2019, ApJ, 885, 34

Yuan, D., \& Nakariakov, V. M. 2012, A\&A, 543, A9 


\section{Appendix A: Thin flux tube model}

In this study, we use a magnetostatic plasma to model the coronal loop of interest. This model accounts for plasma stratification and a static magnetic field. In our case, the loop has a lifetime significantly greater than the timescales of thermal conduction and radiative cooling, we resort to a magnetostatic plasma. This loop have large aspect ratio (ratio between the loop length and radius), we use thin-flux tube approximation (e.g., Ferriz-Mas \& Schüssler 1989) to model a twisted coronal loop. We expand the MHD quantities for a thin cylindrical flux tube with cylindrical coordinates $(r, \varphi, z)$. We consider an axisymmetric magnetohydrostatic equilibrium solution to the MHD equations. We assume the velocity $\boldsymbol{v}=0$ and set all time- and $\varphi$-derivatives to 0 . In particular, we use the following thin flux tube expansion for the loop parameters,

$$
\begin{aligned}
B_{r}(r, z) & =r B_{r 1}(z) \\
B_{\varphi}(r, z) & =r B_{\varphi 1}(z) \\
B_{z}(r, z) & =B_{z 0}(z)+r^{2} B_{z 2}(z) \\
p(r, z) & =p_{0}(z)+r^{2} p_{2}(z) \\
\rho(r, z) & =\rho_{0}(z)+r^{2} \rho_{2}(z) \\
T(r, z) & =T_{0}(z)+r^{2} T_{2}(z),
\end{aligned}
$$

where $\boldsymbol{B}=\left(B_{r}, B_{\varphi}, B_{z}\right)$ is the magnetic field, $p$ is the pressure, $\rho$ is the density and $T$ is the temperature.

In this study, we derive the key equations for a thin and twisted flux. We expand the magnetohydrostatic equations in the radial component, and balance the forces in the flux tube at a certain distance $R(z)$ with an external pressure force $p_{\mathrm{e}}(R(z))$. The latter relationship is their closure relationship for the (otherwise) infinite system of equations. The resulting equations for the thin and twisted flux tube are

$$
\begin{aligned}
\mathcal{R} T_{0} p_{0}^{\prime}+g p_{0} & =0 \\
B_{r 1}+\frac{1}{2} B_{z 0}^{\prime} & =0 \\
p_{2}+\frac{1}{2 \mu}\left(-B_{z 0} B_{r 1}^{\prime}+2 B_{z 0} B_{z 2}+2 B_{\varphi 1}^{2}\right) & =0 \\
B_{z 0} B_{\varphi 1}^{\prime}-B_{z 0}^{\prime} B_{\varphi 1} & =0
\end{aligned}
$$

$p_{2}^{\prime}-p_{0}^{\prime}\left(\frac{p_{2}}{p_{0}}-\frac{T_{2}}{T_{0}}\right)+\frac{1}{\mu}\left(B_{r 1} B_{r 1}^{\prime}-2 B_{r 1} B_{z 2}+B_{\varphi 1} B_{\varphi 1}^{\prime}\right)=0$

$$
p_{0}+\frac{B_{z 0}^{2}}{2 \mu}+R^{2}\left(p_{2}+\frac{B_{r 1}^{2}+B_{\varphi 1}^{2}}{2 \mu}+\frac{B_{z 0} B_{z 2}}{\mu}\right)=p_{\mathrm{e}}
$$

where primes denote the derivative with respective to $z, \mu$ is the magnetic permeability, $g$ is the gravity pointing in the negative $z$-direction, and $\mathcal{R}$ is the universal gas constant divided by the molar mass.

Since the radial expansion is of particular importance in our problem, let us relate it to the magnetic field variables in the problem. The general equation for the flux surface in the $(r, z)$ plane is

$$
\frac{d r}{d z}=\frac{B_{r}}{B_{z}}=\frac{B_{r 1}}{B_{z 0}} \frac{r}{1+r^{2} \frac{B_{z 2}}{B_{z 0}}},
$$

This is not a separable equation. However, when we take $B_{z 2}=0$ as done in Vasheghani Farahani et al. (2010), the equation becomes

$\frac{d r}{d z}=\frac{B_{r 1}}{B_{z 0}} r$

Using Eq. A.8, we find as solution

$R=R^{\star} \sqrt{\frac{B_{z 0}^{\star}}{B_{z 0}}}$

where we use the same $\star$ notation to indicate the value at a reference height $z=0$. Indeed, this equation expresses the conservation of magnetic flux in a flux tube: as the magnetic field decreases, the radius of the flux tube must increase quadratically.

\section{Appendix B: Solution for an expanding loop}

We assume that the loop radius expands exponentially with the height (see also Dudík et al. 2014):

$R=R^{\star} \exp (z / L)$,

For an expansion factor $R\left(z_{\text {top }}\right) / R^{\star}=\eta$, we find $L=z_{\text {top }} / \ln \eta$. In particular, we can consider $\eta=2$ or $\eta=1.5$ for the observations and $z_{\text {top }}=130 \mathrm{Mm}$, then $L=186 \mathrm{Mm}$ or $320 \mathrm{Mm}$, respectively.

From the conservation of magnetic flux (Eq. A.15), we then find

$B_{z 0}=B_{z 0}^{\star} \exp (-2 z / L)$,

Eq. A.8 can be used to find the radial component

$B_{r 1}=\frac{B_{z 0}^{\star}}{L} \exp (-2 z / L)$

and Eq. A.10 can be integrated (following by Ferriz-Mas \& Schüssler (1989)) to determine the $\varphi$-component of the magnetic field:

$B_{\varphi 1}=B_{\varphi 1}^{\star} \exp (-2 z / L)$,

From Eq. A.9, we obtain

$p_{2}=-\frac{B_{z 0}^{2}}{\mu}\left[\frac{1}{L^{2}}+\left(\frac{B_{\varphi 1}^{\star}}{B_{z 0}^{\star}}\right)^{2}\right] \exp (-4 z / L)$,

This equation shows that $p_{2}<0$, and in particular the total pressure $\left(p_{0}+r^{2} p_{2}\right)$ will thus be negative beyond a critical radius.

Let us now assume that we have a constant temperature $T_{0}$ without radial variation $\left(T_{2}=0\right)$. From Eq. A.7, we obtain in this case

$p_{0}=p_{0}^{\star} \exp (-z / H)$,

where we define the scale height $H=\mathcal{R} T_{0} / g$. For a temperature of $T_{0}=0.7 \mathrm{MK}$, the scale height is about $22.8 \mathrm{Mm}$, and this value is increased to $38 \mathrm{Mm}$ if we reduced the gravity by projection.

We then employ Eq. A.12 to obtain external pressure of the tube $p_{e}$ :

$p_{e}(z)=p_{0}^{\star} \exp (-z / H)+\frac{B_{z 0}^{\star 2}}{2 \mu} \exp (-4 z / L)-\frac{B_{z 0}^{\star 2}}{2 \mu} \exp (-2 z / L)\left(\frac{R^{\star 2}}{L^{2}}+\left(\frac{R^{\star} B_{\varphi 1}^{\star}}{B_{z 0}^{\star}}\right)^{2}\right)$.

Given that $R^{\star} / L$ is small, the external pressure usually remains positive. Only when the twist term $R^{\star} B_{\varphi 1}^{\star} / B_{z 0}^{\star}$ is large, then the external pressure becomes negative. Thus, we can find an upper limit for the twist (see, Figure B.1). For an expansion factor of 1.5 (or 2.0), the upper limit of the twist is around 0.65 (or 0.5). These values are compatible with the limits for kink instability. 


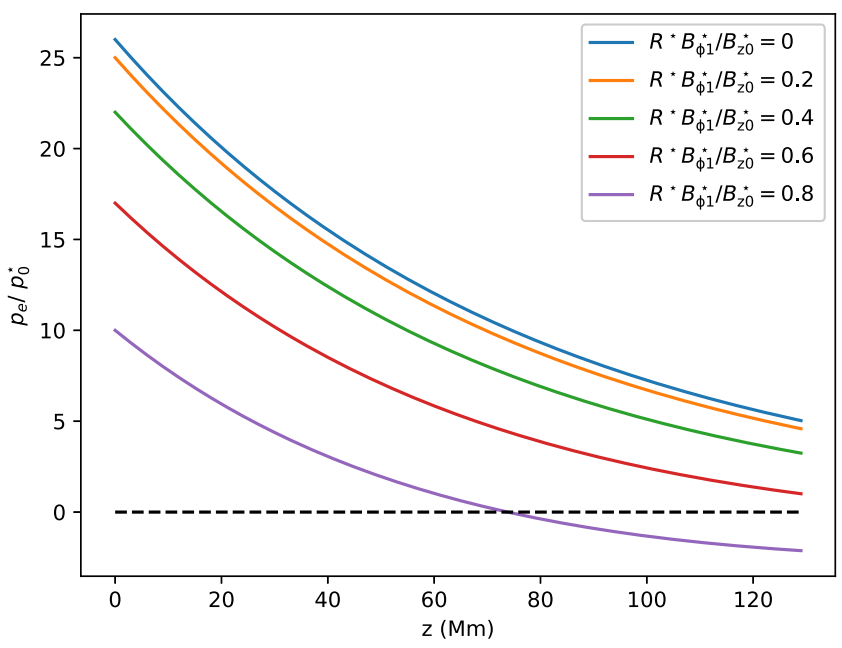

Fig. B.1. Profiles of the gas pressure ratio of the external and internal plasma for various twist applied. 